\title{
Für eine Neubestimmung der Bildungsaufgaben
}

\section{Die Ernährungs- und Verbraucherbildung ist ein unverzichtbares Element einer zukunftsorientierten Grundbildung, welche die notwendige Voraussetzung für die Bewältigung und verantwortliche Gestaltung von Gegenwart und Zukunft schafft. Die zu entwickelnden Lebensführungs- und Lebensgestaltungskompe- tenzen betreffen sowohl die individuelle Ebene, den Sozialverband wie auch die gesellschaftlichen Zusammenhänge.}

$\mathrm{E}$

Von Sigrid Beer

pflichtenden Bildungskanon der allgemein bildenden Schule. Sie steht in der Verantwortung den Bildungsanspruch der Kinder und Jugendlichen durch den individuellen Bildungserfolg einzulösen. Das gilt für alle Schulstufen, Schulformen und Bildungsgänge. Das ist das Credo des Forschungsprojekts Reform der Ernährungs- und Verbraucherbildung in Schulen (Revis), das im Sommer 2005 seine Arbeitsergebnisse vorlegen wird (1).

Der Diskurs über die Elemente einer zukunftsorientierten Grund- und damit Allgemeinbildung muss offensiv und öffentlich geführt werden. Das ist zurzeit nicht der Fall. So besteht die Gefahr, dass der Bereich der Lebenskompetenzen nicht nur nicht angemessen genug berücksichtigt wird, sondern im Gegenteil sogar noch weiter aus den Stundentafeln verdrängt wird, weil eine deutliche Konzentration auf die so genannten Kernfächer erfolgt. Den Erwerb der Kulturtechniken, die unverzichtbar für eine erfolgreiche Lebensfïhrungund Lebensgestaltung sind, zu ermöglichen und $\mathrm{zu}$ sichern, ist eine staatliche Aufgabe.

\section{Devise Prävention}

Prävention muss die Devise auch in der Bildung sein, statt immense Ressourcen für das Kurieren von Bildungsversäumnissen und Fehlentwicklungen aufzuwenden, die die individuellen Lebenschancen und gesellschaftliche Entwicklungen massiv beeinträchtigen. Die Schule muss das Leben ernst nehmen, auf situiertes Lernen setzen und die anwaltschaftliche Vertretung für den Bildungserfolg ihrer Schülerinnen und Schüler in allen Dimensionen übernehmen, auch und gerade in der Ernährungs- und Verbraucherbildung. Von den erfolgreichen Pisa-Nationen zu lernen, heißt wahr- zunehmen, dass dort gesellschaftliche Entwicklungen bezüglich der Bildung von Kindern nicht beklagt sondern behoben werden. Der Funktionsverlust von Familie eventuell sogar mit Schuldzuweisungen versehen wird nicht wie vor einer Klagemauer laut ausgerufen. Ein Unterstïtzungs- und Verantwortungsbündnis macht sich an die Bewältigung der Aufgaben, die sich aus den festgestellten Bildungsdefiziten ergeben. Das gilt für die Verantwortungsübernahme durch die Bildungsinstitutionen von der Elementarbildung an, sowie für die Entwicklung von Bildungsnetzwerken, die auch Elternarbeit und Elternbildung leisten.

Das Forschungsprojekt Revis hat die Initiative ergriffen, in einem großen Kooperationsvorhaben, gemeinsam mit Institutionen ausgewählter Bundesländer, praktikable Vorschläge für eine Reform der Ernährungs- und Verbraucherbildung in allgemein bildenden Schulen zu erarbeiten und diese den zuständigen Länderministerien und Senatsverwaltungen, Lehrplankommissionen sowie den Schulen zur Verfügung zu stellen:

- Bildungsziele, Bildungsstandards und Kerncurriculum, die an ein internationales Bildungsverständnis anknüpfen und an die föderalen Strukturen anschlussfähig sind, ergeben einen praxisorientierten Orientierungsrahmen, um die angestrebte Grundbildung im Bereich der Ernährungs- und Verbraucherbildung zu ermöglichen. Die entwickelten Rahmenvorgaben können zudem von unterschiedlichen Fächern genutzt werden und ermutigen zum fächerübergreifenden Arbeiten, da sie Natur- und Kulturwissenschaft integrieren.

- Ein Portfolio mit Aus- und Fortbildungsprofilen wird als ein Instrument zur persönlichen Leistungs- und Qualifikationsübersicht und Professionalisierung für Lehrerinnen und Lehrer entwickelt.
- Vorhandene Unterrichtsmaterialien werden analysiert und bewertet, inwieweit sie den Kriterien einer zukunftsorientierter Ernährungs- und Verbraucherbildung entsprechen. Daraus werden Prüf- und Entwicklungskriterien gewonnen, die den Lehrerinnen und Lehrern bei der Unterrichtvorbereitung helfen.

- Es wurde eine mobile Esswerkstatt entwickelt, die Schulen ohne Schulküche eine professionelle Sinnes- und Geschmacksbildung sowie Experimente mit Nahrungsmitteln ermöglicht. Eine solche Werkstatteinheit kann auch den vorhandenen Fachraum Küche entlasten.

- Ein zentrales Internetportal wird im Laufe des Projekts entwickelt (2).

- In einer Feldstudie wird die Akzeptanz der Bildungsziele und des Kerncurriculums evaluiert. Dazu werden Lehrerinnen und Lehrer, aber auch Eltern und Schülerinnen und Schüler befragt.

Die Ergebnisse des Forschungsprojekts sollen nicht nur für die Lehrerinnen und Lehrer, sondern auch für Schülerinnen und Schüler und für die Eltern verstehbar, handhabbar und bedeutsam sein. Nur dann kann ein breiter Diskurs über die Bedeutsamkeit der Domäne Ernährungs- und Verbraucherbildung gelingen.

\section{- Das Revis-Haus}

Einen ersten Eindruck vermittelt dazu das RevisHaus der Bildungsziele, das im Folgenden abgebildet ist. Die Bildungsziele bilden das Dach und werden in Schlüsselfragen übersetzt, die die Fenster im Haus darstellen. Mit dem Blick in diese Fenster werden die Kerninhalte der Ernährungsund Verbraucherbildung vorstellbar und erfassbar. In den Schlüsselfragen wird die Grundphilosophie des Revis-Ansatzes gewahrt, den essenden und handelnden Menschen und den Erhalt und die Entwicklung der individuellen und sozio-kulturellen Ressourcen in den Mittelpunkt zu stellen.

\section{Anmerkungen}

(1) Das interdisziplinäre Projekt REVIS wird finanziell unterstützt durch das Bundesministerium für Verbraucherschutz, Ernährung und Landwirtschaft (BMVEL).

(2) www.evb-online.de

\section{Die Autorin}

Sigrid Beer ist freiberufliche Mitarbeiterin im Projekt REVIS und Lehrbeauftragte an der Universität Paderborn. Kontakt: Universität Paderborn, Fakultät NW, FG Ernährung und Verbraucherbildung, Warburger Str. 100, 33098 Paderborn.

E-Mail: sbeer@evb.upb.de 
(c) 20I0 Authors; licensee IÖW and oekom verlag. This is an article distributed under the terms of the Creative Commons Attribution Non-Commercial No Derivates License (http://creativecommons.org/licenses/by-nc-nd/3.o/), which permits unrestricted use, distribution, and reproduction in any medium, provided the original work is properly cited. 